\title{
Gradhiva
}

GRADHIV

Revue d'anthropologie et d'histoire des arts

26 | 2017

En croire ses sens

\section{D'argile, de fleurs et de laine. Catéchismes multisensoriels dans les hauts plateaux boliviens}

of clay, Flowers and Wool. Multisensory Catechisms in the Bolivian Highlands

\section{Bérénice Gaillemin}

\section{OpenEdition}

1 Journals

\section{Édition électronique}

URL : http://journals.openedition.org/gradhiva/3431

DOI : 10.4000/gradhiva.3431

ISSN : 1760-849X

Éditeur

Musée du quai Branly Jacques Chirac

\section{Édition imprimée}

Date de publication : 6 décembre 2017

Pagination : 46-71

ISBN : 978-2-35744-096-8

ISSN : 0764-8928

Référence électronique

Bérénice Gaillemin, «D’argile, de fleurs et de laine. Catéchismes multisensoriels dans les hauts plateaux boliviens », Gradhiva [En ligne], 26 | 2017, mis en ligne le 06 décembre 2019, consulté le 03 janvier 2020. URL : http://journals.openedition.org/gradhiva/3431 ; DOI : 10.4000/gradhiva.3431 



\section{D'argile, de fileurs et de laine}

Catéchismes multisensoriels dans les hauts plateaux boliviens

par Bérénice Gaillemin

Dans les hauts plateaux boliviens, les rezos sont des disques d'argile dont la surface présente différents objets et figurines en argile ordonnés en colimaçon. Chacun des objets transcrit un mot ou une syllabe du texte des prières catholiques en quechua, enseignées pendant le Carême. Les rezos sont détruits à l'issue de la Semaine sainte. Nous proposons ici une approche pragmatique de l'élaboration de ces artefacts à la fois dans leur matérialité et leurs modalités d'usage, qui fait appel aux sens - vue, ouïe et toucher. Sont d'abord exposés les ressorts de cette mnémotechnie et les éléments relatifs au fonctionnement (et dysfonctionnement) sémiotique de ce système, pour montrer ensuite qu'au-delà d'un système scriptural, les rezos correspondent à une miniaturisation des activités quotidiennes et agropastorales des communautés. Enfin, sont analysées l'ensemble des activités organisées pendant le temps rituel, qui va du Carême à la Semaine sainte, marquées par un fort contraste sensoriel. 


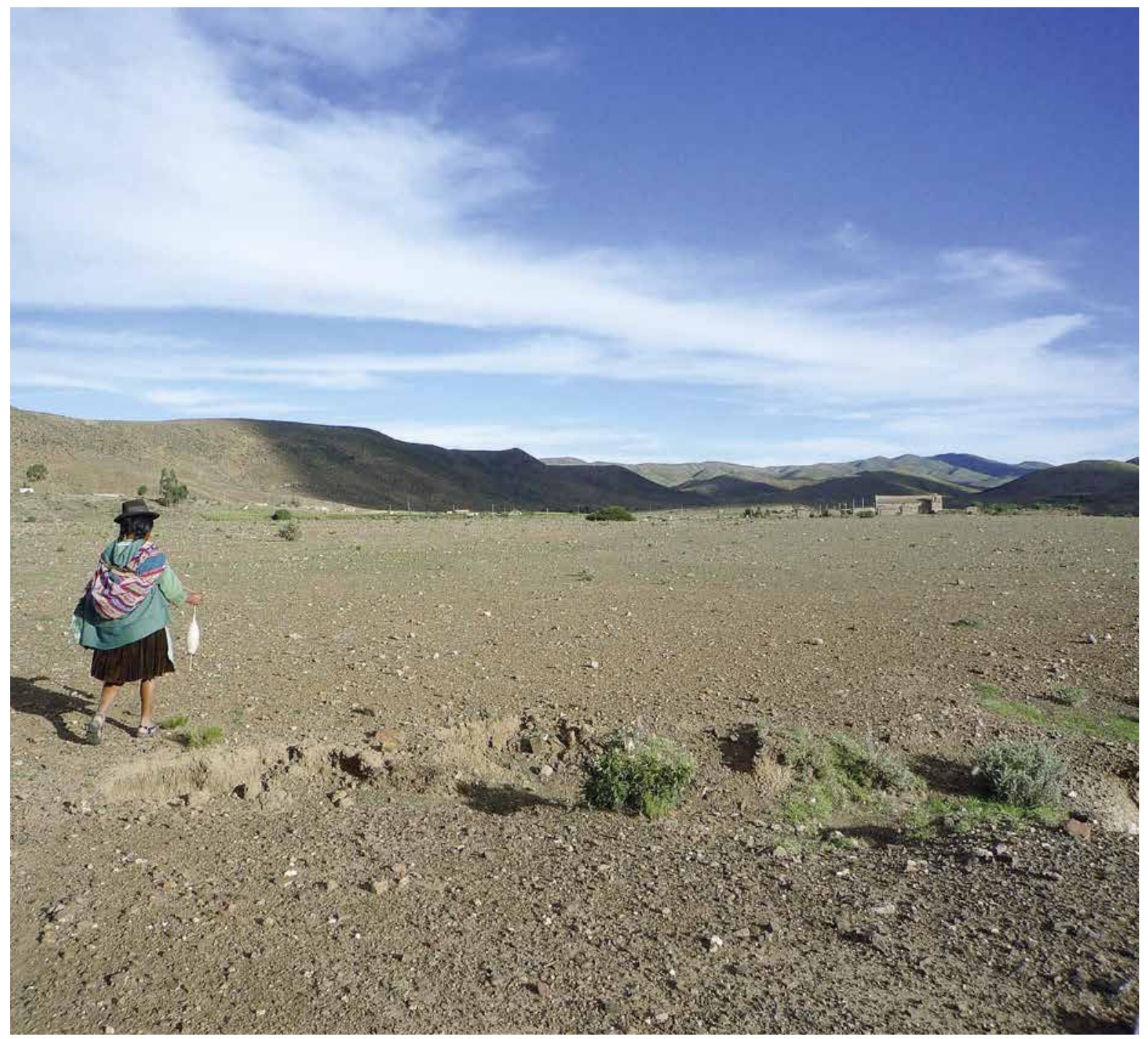

fig. 1

Paysage environnant

de la municipalité de

San Lucas, 2013. Photo

Bérénice Gaillemin. 
En espagnol, un rezo est une prière et rezar correspond à l'acte de prier. Les prières catholiques sont généralement transcrites dans des livres reliés ou des petits catéchismes communément appelés Doctrinas. Mais dans les hauts plateaux boliviens, le mot rezo désigne aussi des disques d'argile modelés sur le sol des églises. Sur leur surface, différents objets et figurines en argile sont ordonnés en colimaçon. Chacun de ces objets correspond à un mot ou une syllabe du texte des prières dont la transmission s'effectue pendant la période de la Doctrina, consacrée à l'enseignement des textes du catéchisme. Chaque année, pendant le Carême, dans plusieurs communautés indigènes situées à moins de $150 \mathrm{~km}$ de la ville minière de Potosi ${ }^{1}$, des doctrineros («maîtres responsables de la doctrine», aussi appelés maestros) enseignent ces prières et textes religieux - parmi lesquels le Notre Père, le Je vous salue Marie et les Dix Commandements dont les autorités locales exigent la mémorisation.

C'est en langue quechua, la langue d'usage, qu'elles sont transmises et mémorisées puis récitées pendant les processions et les veillées clôturant la Semaine sainte, à l'issue de laquelle les rezos sont détruits pour être fabriqués à nouveau l'année suivante.

L'usage des rezos d'argile a vraisemblablement été imposé par le passé par des membres de l'ordre jésuite. Faute de mentions de cette pratique dans les archives, il est toutefois difficile d'en connaître l'époque d'origine. En revanche, il est important de souligner qu'aujourd'hui les autorités ecclésiastiques n'y participent aucunement. Ainsi Padre Santos, prêtre assigné à la paroisse ${ }^{2}$ en avril 2017, s'étonnait de découvrir cette pratique dont il n'avait auparavant jamais entendu parler. En effet, c'est désormais de façon autonome et à l'initiative des autorités indigènes locales que se fait l'enseignement des prières en quechua. Les rezos sont donc associés à une costumbre, une façon de faire qui est propre aux communautés respectant la «tradition". Pour en comprendre le fonctionnement (leur confection, transmission et périodicité), il est nécessaire d'expliquer brièvement le découpage politicoadministratif de la région.

Le municipio (la «municipalité") de San Lucas (32 000 habitants) comprend quinze distritos («districts»), dont chacun réunit à son tour un certain nombre de comunidades ("communautés"). Ces dernières sont parfois regroupées: ainsi le district de Padcoyo comprend un groupe (sub-central) de cinq communautés et un second de six.

\begin{tabular}{|c|c|}
\hline \multirow{4}{*}{ PADCOYO } & Kumuni \\
\cline { 2 - 2 } & Andajava \\
\cline { 2 - 2 } & Tirahoyu \\
\cline { 2 - 2 } & Miska Mayu \\
\cline { 2 - 3 } & Puka Loma \\
\cline { 2 - 3 } & Sivinga Mayu ${ }^{3}$ \\
\cline { 2 - 3 } & Churqui Pampa \\
\cline { 2 - 3 } & Punquina \\
\hline & Monte Pampa \\
\hline & Quiwayllani \\
\cline { 2 - 2 } & Padcoyo \\
\hline
\end{tabular}

1. C'est plus précisément dans la municipalité de San Lucas (dans la province Nor Cinti, l'une des dix provinces du département de Chuquisaca) que se situent les communautés. Les informations et photographies de cet article ont été collectées lors de terrains effectués pendant le Carême en 2011, 2013, 2014 et 2017, pour la plupart dans les communautés de Padcoyo, Tirahoyu et Cinteño Tambo. Ces terrains ont été entamés dans le cadre d'un projet collectif (avec Pierre Déléage et Isabel

Yaya) et ont bénéficié des financements du CNRS et du LabEx TransferS. Je les a poursuivis dans le cadre de deux post-doctorats effectués au LabEx HASTEC (rattachement au CéSor) et au LabEx TransferS (rattachement au Laboratoire d'anthropologie sociale - LAS - et à son équipe d'anthropologie linguistique).

2. Le prêtre réside à San Lucas, village chef-lieu qui compte une église. Il est en charge de l'ensemble de la paroisse, dont les frontières sont identiques à celles de la municipalité.

3. Les communautés don le nom est transposé en gras forment le deuxième sous-groupe du district.
4. Située à 3200 mètres d'altitude, Padcoyo est la communauté d'où provient l'essentiel de l'ethnographie restituée ici. En plus des commentaires dus aux élèves, la plupart des renseignements relatifs au sens des signes ont été fournis par le doctrinero Isidoro Flores, né en 1946 et appartenant à la communauté de Tirahoyu. Connu pour son savoir-faire et sa connaissance de la Doctrina, celui-ci a été élu et engagé pendant une vingtaine d'années, jusqu'en 2015, par différentes communautés du district de Padcoyo. S'il contribue encore parfois à la confection des rezos, il ne souhaite plus enseigner et a transmis son savoir à d'autres membres des communautés, chargés maintenant de leur enseignement annuel. Malgré la rotation théorique des charges, il s'avère en effet que les doctrineros se révèlent être, dans la pratique, les mêmes d'une année sur l'autre. Moyennant finance. un maestro auquel la charge est imposée par sa communauté pourra en effet la déléguer à quelqu'un de plus compétent, moins occupé ou plus catholique que lui. Il est courant également qu'une même charge puisse être endossée par un ou plusieurs membres d'une même famille.

fig. 2

District de Padcoyo et ses onze communautés 4 
5. Une communauté dont le corregidor - la personne élue annuellement à la plus haute fonction politique, avec les kuraka, auxiliar, etc. - appartient à l'Église évangélique ne participe pas à cette organisation rotative des rezos. En revanche, elle prend part à toutes les activités collectives organisées par la communauté ou le district en dehors du domaine religieux.

6. Ces volontaires non rémunérés sont chargés d'apporter de la nourriture (boisson, déjeuner et dîner) au maestro pendant qu'il enseigne la Doctrina. Ils sont aidés par le fiscal (traduit wishkal en quechua), responsable de la discipline rappelant à l'ordre les élèves dissipés en agitant un chicote (sorte de martinet).

7. Je partage ici la définition donnée par Pierre Déléage. Les logogrammes notent des mots, c'est-à-dire des unités lexicales (sémantiques et phonologiques) de la langue quechua. Les phonogrammes notent des unités phonétiques (et non sémantiques) de la langue quechua.

8. Au sujet des rébus utilisés dans le cadre de l'apprentissage du catéchisme à l'époque coloniale au Mexique et dans les Andes boliviennes depuis le début du xx siècle.
9. Les doctrineros connaissent généralement les prières par cœur. Pour les transcrire, ils se servent parfois de cahiers où ils ont préalablement dessiné les objets et

figurines correspondants. Plusieurs autres doctrineros appartenant à des communautés oublieuses de cette méthode recourent à un livre publié en 1988. Celui-ci est l'œuvre du père Juan Miranda et d'un doctrinero aujourd'hui décédé, Eulogio Juárez. Les prières y ont été transcrites à la fois en caractères latins et en images de type Clipart car l'informatisation a entraîné une modification de la sémiotique et l'intégration de nouvelles images (parfois considérées par le père Miranda comme une amélioration).

La circulation de ce livre a modifié la pratique des rezos dans plusieurs communautés dont nous ne traitons pas ici.
Chacun de ces groupes organise annuellement la fabrication et la transmission des rezos: une communauté se charge de recruter, payer et de tout organiser, chacune son tour. Dans un groupe de six communautés ${ }^{\mathbf{5}}$, ce devoir revient donc à la même communauté tous les six ans.

Chaque année, les autorités locales élues annuellement dans leur communauté se réunissent et votent à main levée pour élire le maestro qui devra confectionner les rezos et les enseigner dans les semaines précédant Pâques. Payé par l'ensemble de la communauté, il devra se rendre disponible pendant plusieurs jours, aidé dans sa tâche par des «volontaires » désignés par les mêmes autorités ${ }^{6}$. L'enseignement des prières s'adresse aux membres inactifs des communautés: tandis que les enfants apprennent leurs prières à la sortie de l'école ou le week-end, les personnes âgées mémorisent le catéchisme pendant la journée. Débutant en moyenne trois semaines avant la fin du Carême, l'apprentissage s'intensifie à l'approche des célébrations de Pâques.

Comme on l'a dit, l'enseignement se fonde sur l'usage de textes écrits au moyen d'objets et de figurines modelées en argile, le tout disposé sur un disque lui-même placé sur le sol. Ces objets et figurines peuvent être considérés comme les signes d'un système scriptural à la fois pictographique et tridimensionnel. Ainsi, la reproduction miniature en argile d'une pierre à moudre transcrit le verbe «moudre» et la présence d'une fleur indique le nom de celle-ci. Une figuration de la Vierge Marie fournit le nom de cette dernière, tandis que la figuration d'un chien évoque le son de son aboiement.

Ces signes sont littéralement lus les uns après les autres et transposent des mots et des syllabes. Bien que leur apparence soit singulière puisqu'il s'agit d'objets, ce sont bien des logogrammes ou des phonogrammes ${ }^{7}$ (transcrivant une syllabe, plus souvent deux) et non des symboles disposés de façon arbitraire comme le seraient par exemple les éléments d'une crèche. Leur emploi peut se fonder sur l'homophonie entre deux termes: la prononciation à haute voix de la valeur d'un signe renvoie alors à une toute autre réalité sémantique que celle de l'objet concerné. Le nom de la fleur ou les phonèmes contenus dans l'onomatopée qui correspond à l'aboiement d'un chien permettent donc de transcrire les sons d'un autre mot: c'est ce que l'on nomme communément un rébus ${ }^{8}$. Ainsi, les homophonies jouent sur la proximité entre les significations et les sons, donnant l'occasion d'associer les registres sémantique et phonétique. Les habitants étant généralement bilingues quechua-espagnol, les jeux se compliquent encore lorsqu'ils profitent d'une homophonie entre les deux langues.

II s'agit ainsi d'une écriture complexe qui suit plusieurs étapes. En premier lieu, tandis qu'ils existent au préalable sous leur forme alphabétique, les textes des Doctrinas imprimées ${ }^{9}$ sont traduits en objets : cette méthode implique donc le passage d'un système sémiotique à un autre. II ne s'agit pas, en effet, de l'écriture d'un texte ayant au préalable circulé uniquement oralement mais de ce que Pierre Déléage appelle une «écriture secondaire» (Déléage 2013: 169). La disposition des signes les uns après les autres est en cela influencée par l'écriture alphabétique. En deuxième lieu, le «scribe" tout comme le «lecteur » doivent opérer une synthèse lors du passage du registre sémantique au registre phonétique: il s'agit de déterminer, après transcription et décryptage, le vocabulaire dont il est réellement question dans la prière parmi tous les mots qui lui sont phonétiquement proches. Enfin, on note un perpétuel va-et-vient entre les langues utilisées pour prier 
(textes mêlés de quechua et d'emprunts castillans) et pour déchiffrer l'écriture (jeux homophoniques jonglant avec deux langues). Cette alternance illustre non seulement les aptitudes linguistiques des habitants des communautés, mais révèle aussi l'ancienneté des prières mémorisées, traduites en quechua par des missionnaires dès le $\mathrm{XV}^{e}$ siècle ${ }^{10}$. Et hormis quelques variations, les textes sont globalement les mêmes d'une communauté à l'autre ${ }^{11}$.

Interroger les façons dont les maîtres et les élèves expérimentent cette méthode permet de mieux en comprendre les enjeux. Certes, les enfants sont parfois encore trop jeunes pour connaître les rudiments de l'alphabet et les personnes âgées peuvent n'avoir jamais appris à lire et à écrire. Pourtant, si on a recours à cette méthode, ce n'est certainement pas à défaut d'alphabétisation ${ }^{12}$ mais bien pour conjuguer divers aspects esthétiques et délivrer ce qui pourrait s'apparenter à une «leçon de choses». Nous nous demanderons dans quelle mesure une approche pragmatique du cadre spatio-temporel de la leçon, associée à une analyse des objets utilisés pour transcrire des textes religieux, peut nous renseigner sur la façon dont ces communautés mêlent concrètement pratiques religieuses et vie quotidienne. Au-delà d'une simple analyse sémiotique, l'usage d'un vocabulaire religieux fait d'objets tangibles eux-mêmes inscrits dans un espace, permet de matérialiser et de transmettre sur le plan sensoriel à la fois des manières de croire, des façons de reproduire ou d'articuler un texte et enfin des modes de penser, d'éprouver, de ressentir et, plus généralement, d'être au monde.

Je présenterai dans un premier temps les ressorts de cette mnémotechnie tout en fournissant des éléments relatifs au fonctionnement (et dysfonctionnement) sémiotique de ce système. Je montrerai dans un deuxième temps qu'au-delà d'un système scriptural, l'élaboration des rezos peut être comprise comme une miniaturisation des activités quotidiennes et agropastorales des communautés. Enfin, il sera question de l'ensemble des activités rituelles organisées entre le Carême et la Semaine sainte, une période pendant laquelle les cinq sens sont particulièrement sollicités.

\section{Apprendre son caté: en images et en 3D \\ Mémoriser, spatialiser, vocaliser, reconnaître et apprendre}

La méthode d'enseignement des rezos en argile permet avant tout de retenir par cœur un corpus de textes religieux. Les ressorts cognitifs utilisés s'apparentent à ceux de mnémotechnies fameuses semblables aux arts de la mémoire décrits depuis l'Antiquité (Yates 1966). La mémorisation des textes, on le verra, peut être fondée sur la répétition et la musicalité, mais elle convoque également des images ${ }^{\mathbf{1 3}}$ disposées dans des lieux. Cette association concrète images-lieux, connue en Europe sous l'expression «palais de la mémoire ", s'effectue à deux niveaux: chaque image s'inscrit dans un espace délimité (le disque d'argile), lui-même intégré à un espace plus grand, celui de l'église ou du bâtiment collectif de la communauté choisi pour dispenser la leçon ${ }^{14}$.

Cette méthode semble ainsi inclure, à différentes échelles, une dimension spatiale. L'élève, d'un coup d'œil, embrasse l'ensemble du texte qu'il doit apprendre.
10. La Doctrina publiée après le III ${ }^{\circ}$ Concile de Lima (1585) fait aujourd'hui encore autorité dans ces communautés bien que les doctrineros n'en possèdent pas d'édition et que ce soit plutôt de mémoire qu'ils savent les textes.

11. La question de la variation lexicale, syntaxique et sémiotique n'est pas le propos central de cet article. II est néanmoins important de souligner que, malgré les exemples ici choisis, toutes les communautés ne transcrivent pas exactement les textes de la même façon.

12. Excepté les plus jeunes et les plus vieux, la majorité des élèves, âgés de 10 à 14 ans, est parfaitement alphabétisée.

13. On utilisera

indistinctement le terme d'image pour parler de tous les petits objets ou figurines tridimensionnelles renvoyant à un mot.

14. Toutes les communautés ne disposant pas d'église ou de chapelle (comme c'est le cas de Padcoyo, Cinteño Tambo et Palacio Tambo), l'enseignement de la Doctrina peut avoir lieu dans un bâtiment annexe situé dans le hameau (pour les plus petites communautés, comme Tambo Moq'o), dans un petit édifice situé aux alentours d'une église abandonnée (communauté de Tirahoyu) ou dans une ancienne salle d'école (communauté de Puka Loma). 


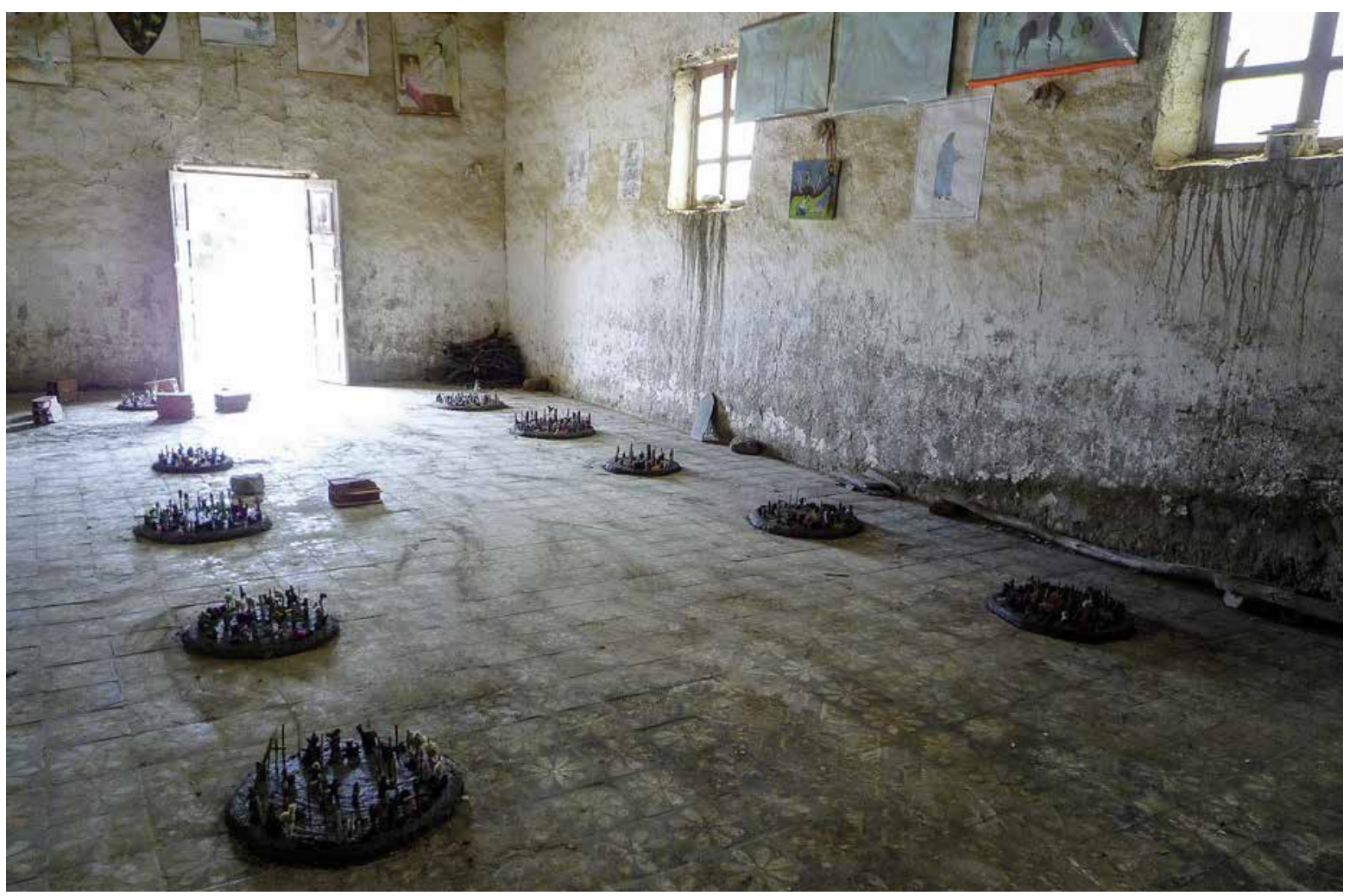

fig. 3

Les dix prières à mémoriser.

La première est le Notre Père, située à gauche de l'entrée de l'ancienne église de la communauté de Padcoyo, 2017. Photo Bérénice Gaillemin.
La dimension à la fois visuelle et spatiale lui permet aussi d'expérimenter les textes de façon originale. Si le texte était transcrit au moyen de l'alphabet, il lui faudrait tourner les pages. À l'oral, il devrait attendre un certain laps de temps avant d'écouter la prière dans son intégralité et en saisir éventuellement le sens. Si ces objets disposés en spirale ne fournissent donc pas directement la clé de leur signification, leur nombre, leur forme, leur orientation et leur taille donnent en revanche des indices sur la longueur de la prière, sa forme de vocalisation et, d'une certaine façon, son contenu. En effet, un disque de plus grand diamètre contiendra davantage de signes, soit davantage de mots ou de syllabes. Un élève est donc directement en mesure d'évaluer la longueur d'un texte. L'absence de signes de ponctuation est par ailleurs l'expression concrète de sa vocalisation: quasiment dans un seul souffle, il sera lu et relu jusqu'à être mémorisé. À la façon d'un texte psalmodié, la prière est sans cesse répétée, prise dans le tourbillon de la spirale en écho à une prononciation ininterrompue et renouvelée à chaque fois que le centre du disque est atteint.

En effet, pour enseigner, le doctrinero entame seul la lecture à voix haute du premier mot: Yayayku («notre père»); les élèves répètent ensemble yayayku. Puis les mots suivants s'enchaînent, hanaqpachakunapi («aux cieux»); hanaqpachakunapi, répondent de façon identique les élèves. Kaq, sutiyki («qui êtes, [que] ton nom»), much'asqa kachun ("soit sanctifié»), etc. C'est ainsi, par cette lecture hachée et continuellement répétée, que le texte est peu à peu mémorisé. Dès que le maître a fini, il reprend l'exercice: Yayayku (seul), yayayku (les élèves), etc. Et tout en prononçant, il désigne chacun des objets et des figurines à l'aide d'une baguette que tous ensemble regardent en même temps qu'ils vocalisent le texte. 
À première vue, certains objets suggèrent aussi le contenu du texte. Par exemple, d'un coup d'œil rapide, l'élève reconnaît aisément le Confiteor, car à plusieurs endroits un objet - en l'occurrence une grosse boule de laine non tissée accrochée à un bâtonnet - renvoie à la notion du péché, terme "péché» qui revient fréquemment tout au long de la prière («moi pécheur... j'ai péché, j'ai péché, j'ai beaucoup péché»).

Détournant le regard du disque pour prendre un peu de hauteur, l'élève comme le maître peuvent également associer chacune des prières à un endroit donné. En effet, les disques obéissent à une disposition précise respectant une sorte de parcours imposé. Le chemin d'apprentissage débute toujours par les prières principales, se poursuit par des textes plus fournis comme celui du décalogue et se termine par des textes plus longs et complexes comme celui des quatorze articles de la foi. Dans la mesure où les textes doivent être mémorisés dans un certain ordre, des groupes sont répartis selon leur niveau de connaissance autour des rezos dont le nombre varie de trois à dix en fonction de la taille du lieu d'enseignement ${ }^{15}$. D'un seul regard, le maître évalue la progression de l'ensemble de ses élèves: un petit groupe reste devant le Notre Père pendant plusieurs jours tandis que d'autres enfants plus grands ou des personnes âgées plus zélées avancent d'un texte à l'autre avec plus d'aisance. C'est après une sorte d'examen durant lequel l'élève lit seul le texte en spirale que le doctrinero l'autorise à passer au disque suivant. Venant de passer des heures, parfois des jours à mémoriser un disque, l'élève change de place en exprimant sa joie. Puisqu'il va de soi que les prières précédentes sont sues de mémoire, enfants comme adultes restituent leur connaissance en précisant le dernier texte qu'ils sont parvenus à retenir: "Je sais jusqu'au Credo", m'explique Carmen, âgée de 35 ans. Bien qu'elle soit la femme du maestro désigné en 2017, ses souvenirs se limitent à quatre prières dont elle conserve la mémoire depuis son enfance. C'est donc en réactualisant l'ordre des prières que les anciens élèves se rappellent combien de textes ils ont appris. En contexte, ils montrent du doigt le rezo auquel ils se sont arrêtés et éprouvent ainsi par leur corps leur progression, se situant aisément au sein du livre - sur le sol de l'église - qu'ils sont en train de lire et d'apprendre.

\section{Métaphores, métonymies et gestes exemplaires}

La disposition, la taille et l'ordre interviennent dans la mémorisation de ces textes, mais aussi dans l'évaluation de leur contenu sémantique et leur prononciation. Par ailleurs, les objets et figurines représentent des personnages, des noms communs, des verbes ou des concepts en recourant à la métaphore, à la métonymie et à d'autres tropes qui permettent d'exprimer une chose dans les termes d'une autre.

Parmi les personnages figurés, celui de la Vierge se distingue car elle porte un morceau de textile de couleur rouge ou verte en guise de manteau. Cette représentation stylisée et d'apparence modeste de la mère de Dieu est en réalité plus élaborée que les autres car elle comprend plusieurs matériaux (argile et textile). Son iconographie, réduite à ce seul attribut textile, permet cependant aux élèves les plus jeunes de la reconnaître rapidement.

Un exemple semblable est celui de la plume qui, plantée dans l'argile, se lit comme le "Saint-Esprit»: renvoyant à la colombe par métonymie, l'objet permet de transcrire le nom d'une des trois personnes de la sainte Trinité.
15. La connaissance du doctrinero peut elle aussi influer sur le nombre de textes transcrits. En 2017 le doctrinero Javier Soto

(de la communauté de Quiwayllani) ajoute un onzième rezo aux dix que lui a transmis Isidoro Flores. II s'agit du Bendito, une prière comprenant un nombre important de termes en castillan. Comme elle est prononcée avant les repas, à la façon du Bénédicité,

la communauté lui en a réclamé l'élaboration et la transmission. 
16. Sans vouloir surinterpréter cette métaphore et y ajouter un sens dont les habitants ne m'ont pas fait part, soulignons néanmoins une éventuelle analogie initiale entre l'objet utilisé aujourd'hui pour désigner le "péché » et le pensum qui, en latin, signifie littéralement un «poids ». Devenu synonyme de punition, le pensum désignait, dans l'Antiquité, le poids de laine que les esclaves devaient filer quotidiennement.

17. On notera également que dans la religion préhispanique l'acte d'adoration s'exprimait par un baiser envoyé par un mouvement des mains en direction de l'objet d'adoration (César Itier, communication personnelle).

\section{ci-contre}

fig. 4

En 2017, la nouvelle doctrinera de Tirahoyu enseigne un rezo à l'aide de sa baguette. L'ancien doctrinero (debout à sa droite) lui explique la valeur de certains signes. Photo Bérénice Gaillemin.

fig. 5

Assis sur des pierres, les élèves suivent du regard la baguette du maestro communauté de Tirahoyu, 2013. Photo Bérénice Gaillemin
C'est suivant ce processus que la boule de laine exprime le concept de péché. Matérialisé par un bâtonnet, l'homme porte ce lourd fardeau de laine dont il se délestera lors de la confession: "C'est quelque chose qui pèse, quelqu'un qui a beaucoup de problèmes ", m'expliquent les élèves. L'objet semble aussi véhiculer le sens initial du terme quechua hucha, qui désignait à l'origine une responsabilité, une obligation non encore acquittée. Dans des communautés agropastorales où l'élevage des brebis et le tissage de la laine sont des activités de subsistance primordiales, il n'est en effet pas surprenant que la charge du péché, au propre comme au figuré, soit transposée de manière métaphorique au moyen d'une matière que l'on porte sur les épaules de la même façon qu'on a l'obligation de la transformer ${ }^{16}$. Par la confession et/ou son tissage, le pénitent se délestera du lourd fardeau ou le travaillera pour le convertir en un textile ordonné et plan. Notons d'ailleurs que l'action d'aplanir a été choisie par les religieux missionnaires du XVle siècle pour traduire le verbe "pardonner» (pampacha-, "aplanir, niveler, pardonner»). On en retrouve une transposition tridimensionnelle dans le rezo correspondant à la prière du Notre Père, où un personnage modelé en position quasi allongée nivèle le sol en guise de pardon.

L'usage du dispositif dans son entier fournit donc des repères à la fois spatiaux et sensoriels qui permettent aux individus impliqués de se situer dans leur apprentissage. Au-delà même de l'iconographie, il est possible de parler de l'acquisition d'un savoir métalinguistique dans la mesure où un sens concret est donné à de nombreux concepts et que l'ensemble du processus joue sur la représentation, la symbolique, l'imaginaire et mobilise les ressources de l'empathie. Le catéchumène se retrouve en effet en position de contempler la Vierge et le Christ crucifié mais, par un effet de miroir, il est également amené à adopter à son tour les attitudes de certaines figurines. L'image d'un personnage agenouillé avec les mains jointes invite par exemple à reproduire ce geste lors de la messe. Par ailleurs, le verbe "sanctifier, louer" - utilisé notamment dans le Je vous salue Marie ou le Notre Père - coïncide avec un groupe de deux personnages se donnant un baiser (much'a-, "embrasser», et much'ayku-, "adorer»). Lorsque je leur demandais le sens de ces figurines, les enfants reproduisaient cette action de grâce destinée à la Vierge en esquissant un baiser. Par l'intermédiaire du rezo vu et appris, ils incorporaient donc un sentiment religieux subtilement inculqué ${ }^{17}$.

Ceci dit, on notera que le recours à ces symboles ne s'assortit d'aucune exégèse de la part du doctrinero mais seulement d'une verbalisation du texte, car c'est un moment uniquement consacré à la prière. Le reste de l'année, les enfants n'ont que très peu l'occasion de se rendre à des classes de catéchisme. Rares sont en effet les catéchistes (catequistas) suppléant aux tâches que le prêtre et le diacre du chef-lieu peinent à accomplir seuls (rappelons que l'ensemble de la municipalité compte cent dix communautés, dispersées sur plus de $\left.4000 \mathrm{~km}^{2}\right)$. Ainsi, tandis que les classes de catéchisme seraient l'occasion de donner des explications plus fournies, l'usage des rezos reste en réalité l'un des rares moyens de voir des images et d'accéder aux textes religieux. À l'église, et le reste du temps, on ne trouve en effet aucune peinture ou sculpture. Hormis quelques ex-voto et dessins effectués par les enfants, les images sont donc quasiment absentes. Et dans les quelques livres qui circulent, il y a si peu d'images que les figurines d'argile 

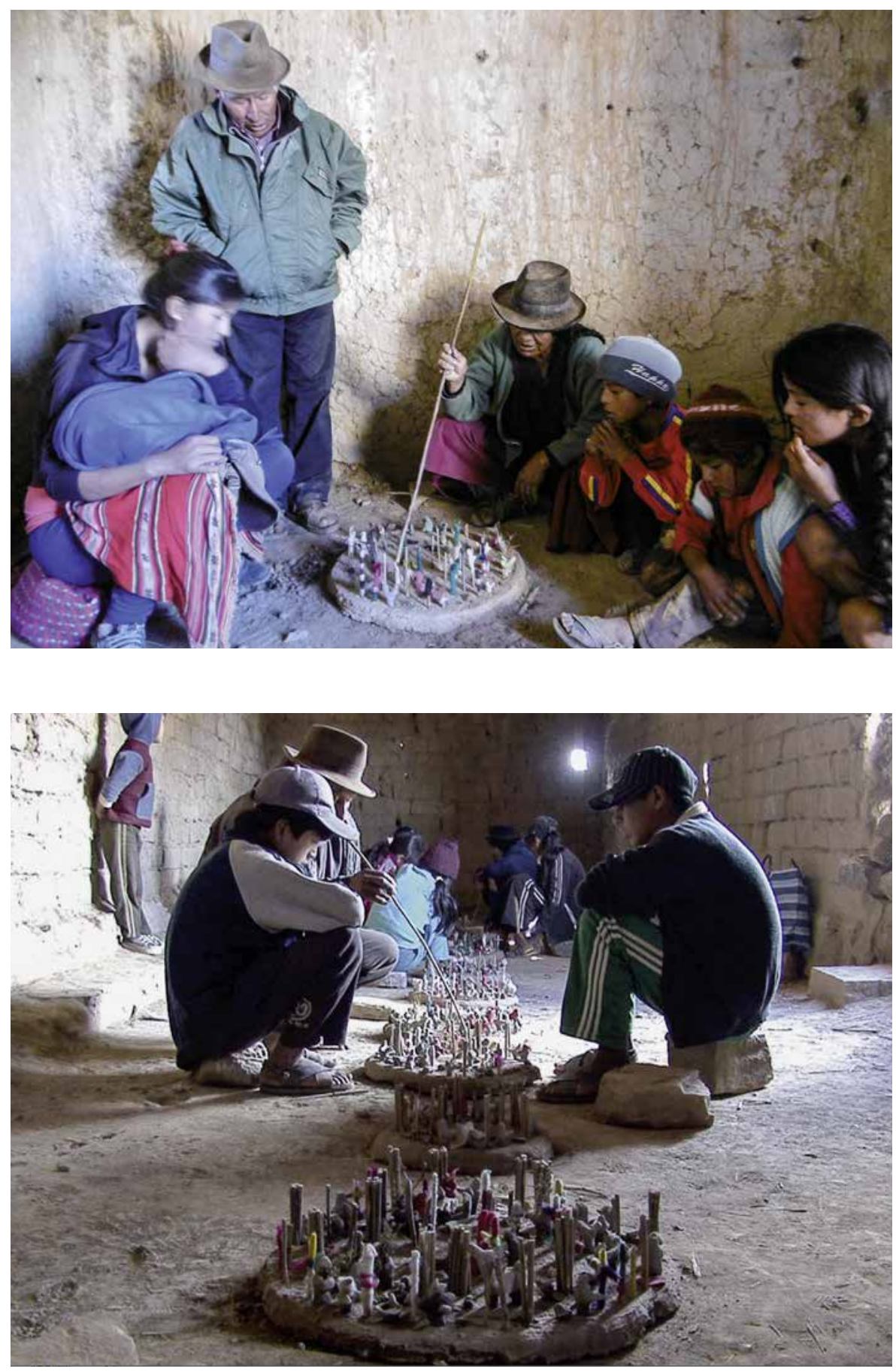
demeurent des référents exceptionnels à l'iconographie religieuse. L'usage de ces symboles aux allures parfois rudimentaires fournit donc aux catéchumènes un enseignement de base, difficile à acquérir autrement.

\section{Jeux de mots et confusions}

Mais plutôt que d'en dépeindre les atouts pédagogiques et leurs effets sur la foi, soulignons malgré tout que ces disques d'argile sont loin de délivrer un message orthodoxe. Par exemple, la particule exprimant l'impératif négatif utilisée lorsqu'il est demandé à Dieu de «ne pas nous laisser tomber dans la tentation ", est ama en quechua. À cause de sa proximité phonétique avec le verbe «aimer» en espagnol (amar), cette particule est transcrite au moyen d'un homme tenant un bébé dans les bras. L'ensemble finit donc par renvoyer à l'amour plus qu'à l'interdiction et la figurine déclenche d'ailleurs plutôt le rire que la crainte. De même, un peigne (peine en espagnol) transcrit les premiers phonèmes du nom du sacrement de la penitencia («pénitence») et une petite calebasse appelée maté ceux du matrimonio.

Tandis que certains signes peuvent parfois jouer sur un registre à la fois sémantique et phonétique, les jeux homophoniques restent la plupart du temps déconnectés de toute réalité religieuse: on n'utilise pas spécialement de peigne lorsqu'est accompli un acte de pénitence et on ne boit pas particulièrement dans une calebasse lors des mariages. Parce qu'ils sont a priori sources de confusion et contredisent les «vertus" associées aux exemples exposés plus haut, ces mélanges de sens et faux-amis posent donc forcément des questions en termes pédagogiques.

L'enseignement didactique des attitudes dévotes et principes religieux à adopter, juxtaposé à un florilège de jeux de mots, est donc a priori tout à fait incongru. Souvent d'ailleurs, les élèves ne comprennent pas tout ce qu'ils mémorisent. Lors des récitations, les phrases sont peu articulées et les archaïsmes de la langue du Xvle siècle sont appris et répétés sans pour autant être saisis. Saint Michel, San Miguel, devient San Mercon ou San Mercanfi, tandis que Ponce Pilate, Poncio Pilato, demeure une image acoustique abstraite à laquelle seule une assiette (homophonie entre pilato et plato, “assiette») est associée. Un même signe peut par ailleurs être différemment interprété par le maître ou les élèves, et les explications diffèrent aussi parfois d'une communauté à l'autre. Ainsi un morceau de tissu (pour dire la «terre», pacha) signifie pour les uns le vêtement, p'acha, et pour d'autres les pages d'un livre dont on m'explique qu'il compte cent (pachak) pages. Aussi, fréquemment les enfants se disputent-ils pour expliquer le sens de tel ou tel objet: «Mais non! Tu te trompes, ça c'est du maïs grillé, hank'a » ou lorsque je leur demande de m'éclairer sur le sens d'une figurine, et bien qu'ils sachent lire globalement l'ensemble, ils se sentent désarmés et en appellent à leur maître: Maestro, imataq kay?, «Maître, qu'est-ce que c'est, ça? ». Les compétences des maestros eux-mêmes sont variées. Il arrive que certains, ayant appris récemment et parfois à la va-vite pour pouvoir accomplir leur charge, interrogent leurs aînés afin de comprendre la lecture de certains signes. Ainsi, en 2017, dans la communauté de Tirahoyu, la doctrinera Antonia Moscosos profitait de la présence d'Isidoro Flores, doctrinero reconnu du district, pour lui demander le sens de certaines figures qu'elle se trouvait en peine de transmettre. 
Enfin, l'inclusion d'objets dont le référent s'est parfois perdu avec le temps est également courante. Dans ce cas, ni le maestro en charge, ni celui qui lui a transmis son savoir ne savent exactement à quoi ils renvoient. C'est par exemple le cas des petits artefacts élaborés pour se référer à saint Pierre et saint Paul: il m'a fallu plusieurs années pour en comprendre le sens. Pour mentionner ces deux saints, deux groupes de bâtonnets sont attachés ensemble par un brin de laine sur lequel est fichée de la laine rose ou rouge. Les deux bâtonnets figurent des hommes qui, par le passé, à pied ou à cheval, et dans une sorte de joute rituelle parfois appelée chiveada, tiraient de côté et d'autre le cadavre d'un chevreau en essayant de faire tomber l'adversaire. La chiveada a marqué l'enfance d'Encarnación, 70 ans, qui se souvient de la frayeur qu'elle lui procurait et de la foule réunie pour les fêtes de saint Pierre et saint Paul. Cette joute n'ayant quasiment plus lieu depuis les années 1950, la signification de ce signe tend aujourd'hui à se perdre pour les élèves comme pour leurs maîtres.

Compte tenu des incongruités liées aux rébus ou de l'emploi de références tombées en désuétude, quel est donc l'enjeu de cette pratique et, surtout, que révèle finalement l'importance que les communautés y accordent?

\section{Appréhender un monde en miniature: couleur, forme, texture, goût et senteur}

Les signes de ces écritures en rébus ne sont ni le produit d'un simple syncrétisme, ni l'héritage colonial d'une dissimulation de pratiques religieuses précolombiennes vis-à-vis des religieux européens. Ils proviennent d'un environnement naturel au sein duquel les habitants vivent, travaillent, croient et pensent ${ }^{\mathbf{1 8}}$. Leur forme, leur couleur, leur orientation spatiale et la matière dont ils sont faits traduisent la vision du monde de leurs concepteurs et utilisateurs. Et à l'instar d'un système linguistique, l'étude de la façon dont cette sémiotique s'intègre à un contexte plus global, donne à voir la façon dont une société organise ses concepts (Lakoff et Johnson 1985; Dehouve 2011 et 2014).

Il apparaît tout d'abord que la logique sous-jacente présidant à l'élection de ces signes à première vue hétéroclites tient à l'usage d'éléments naturels et d'objets indissociables des activités quotidiennes des habitants. En effet, les populations de la région, qui vivent dans un extrême dénuement, subsistent grâce à l'agriculture et à l'élevage. Les principales cultures étant le maïs, le blé, la pomme de terre, la fève et la pêche, les cheptels se composent exclusivement de chèvres et de moutons. Pour enseigner ou apprendre la doctrine chrétienne, les personnes se réunissent donc dans des bâtiments situés dans des communautés villageoises entourées de champs et de montagnes.

Les rezos semblent ainsi ramasser en miniature l'ensemble des activités qui se déploient à taille humaine et naturelle hors des murs de l'édifice. Tels des géants, les catéchumènes se penchent sur les multiples éléments qui évoquent leur propre environnement immédiat.
18. Précisons que le système scriptural employé pour les rezos dans la municipalité de San Lucas reste cantonné à la transcription des prières catholiques: il s'agit d'une écriture "attachée " qui ne transcrit qu'un corpus limité - et dans ce cas, clos de discours (Déléage 2013). Hormis la tentative avortée dans les années 1950 de transcrire d'autres types de textes (hymne national bolivien en quechua par le prêtre de la paroisse, Miranda Rivera 1958), aucun autre n'a été, depuis, transcrit de la sorte. Dans les communautés comme dans le chef-lieu, tout texte est reproduit en caractères latins et la plupart du temps en langue castillane. 


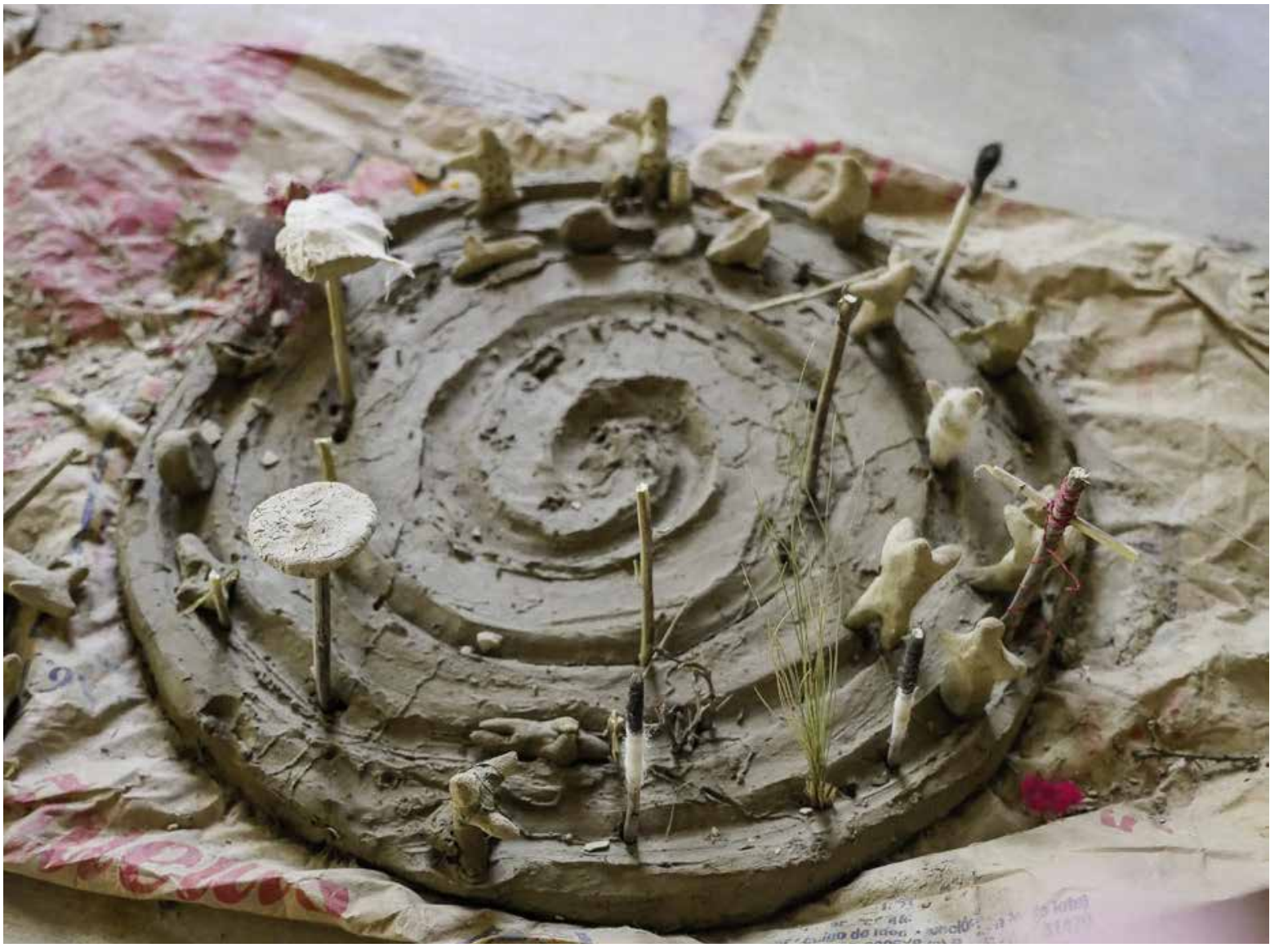

fig. 6

C'est sur une spirale

au sillon plus ou moins

creusé que s'agencent

les objets et figurines,

communauté de Palacio

Tambo, 2015. Photo

Pierre Déléage. 


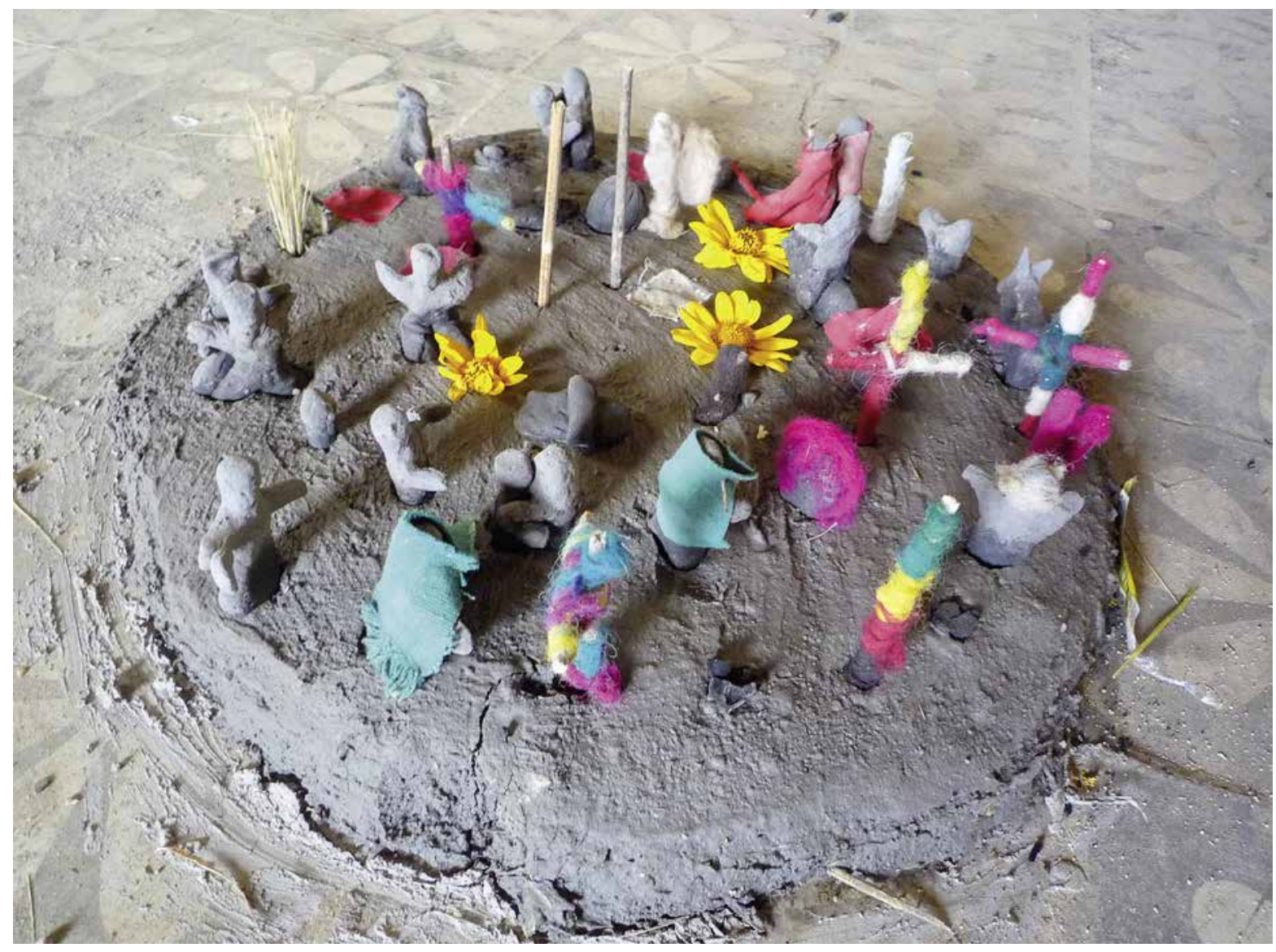

fig. 7

Je vous salue Marie,

communauté de Padcoyo,

2014. Photo Bérénice

Gaillemin. 
19. Notons que le nom de la pêche provient de l'espagnol durazno et se prononce aujourd'hui rurasnu. Les deux premières syllabes sont donc identiques à celles du verbe "créer".

20. Je remercie à nouveau César Itier pour les précisions apportées à ces traductions.

\section{Modeler, semer, récolter, moudre}

Commençons par l'évocation de l'habitat et de l'agriculture. Le disque ainsi que la plupart des figurines qui y sont déposées sont formés d'argile fraîche. Pour le socle, cette dernière est même mélangée à de la paille, comme le sont les briques d'adobe utilisées pour édifier fours, maisons, chapelles et églises. Sur ce socle - et comme dans les champs - de fins sillons sont tracés en surface afin que les figurines puissent y être déposées. Le fruit des travaux agricoles, et en particulier le maïs, est également utilisé pour transcrire certains mots ou suffixes. Le nom de ses grains indique certains sons, et l'objet servant à les réduire en poudre ou en pâte revient tout au long des prières. En effet, une pierre à moudre miniature (modelée en argile) se révèle utile pour écrire les sons contenus dans la marque du pluriel agglutinée aux substantifs de la langue quechua (qhuna-, «moudre», proche de -kuna, suffixe du pluriel).

De la même façon, un noyau de pêche est placé parmi d'autres objets pour évoquer la période du Carême car c'est précisément à cette époque de l'année que sont récoltées les pêches dans toute la région. Or la pêche, fraîche ou pelée puis séchée au soleil (donnant un fruit sec appelé mokochinchi qui reprendra tout son volume une fois associé à de l'eau), fait annuellement l'objet d'une grande fête au chef-lieu de la municipalité. En 2017, celle-ci a eu lieu exactement trois semaines avant Pâques.

Mon hypothèse est que cet objet suggère non seulement une temporalité mais rappelle aussi une restriction alimentaire: le Carême est en effet une période de maigre où les fruits sont préférés à d'autres aliments, et employer cet objet revient à convoquer le sens du goût en même temps que celui de la vue. En établissant ainsi l'analogie entre une sensation gustative et une période temporelle, l'usage d'un simple noyau de fruit permet d'activer une série d'analogies en ricochets, ressenties physiquement chaque année. On notera aussi que le noyau de pêche est utilisé à la fois pour évoquer cette période et transcrire le verbe «faire, créer». L'action de travailler la terre est donc associée plus généralement à ce qui a trait à la création - en l'occurrence la création par Dieu du ciel et de la terre - tandis que le fruit, ruru en quechua, est aussi phonétiquement proche du verbe «créer», rura- ${ }^{19}$.

\section{Animaux, plantes, fleurs et laine}

L'élevage des moutons et le travail de la laine sont des activités essentielles à la survie de ces communautés. On les retrouve en filigrane dans cette sémiotique complexe.

Ainsi, les dents des animaux servent à transcrire phonétiquement «Christ» (Cristo en espagnol, proche de kiru, «dent» en quechua). Les objets qui se réfèrent aux plantes sont également nombreux. Dieu «tout-puissant», llapa atipaq (littéralement «qui peut tout»), est signifié à l'aide d'une herbe llapa, consommée quotidiennement par les animaux. Le «fils» de Dieu, churi, est inscrit par le biais du rameau d'un arbre dont le nom possède les mêmes sonorités (churki). De même, pour dire «l'éternité » de la vie à la fin du Credo, le doctrinero fiche dans l'argile deux belles plantes qui évoquent l'idée de «croissance» des plantes. En effet, le terme choisi par les missionnaires du $\mathrm{XVI}^{\mathrm{e}}$ siècle pour exprimer en quechua le concept d'éternité est wiñay, dont le sens premier est «croissance» mais qui signifie aussi «génération » et, comme adverbe, «des générations », donc «très longtemps ${ }^{20}$ ». 
Au milieu de toutes ces plantes vertes consommées par les animaux, la fleur jaune a sans doute un statut particulier. Elle est utilisée pour transcrire le mot «nom», celui des sept sacrements lorsqu'ils sont énumérés: «le premier, son nom est le baptême», «le deuxième, son nom est pénitence», et ainsi de suite. Cette même fleur jaune sert à transcrire le nom sanctifié de Dieu $^{\mathbf{2 1}}$ dont il est question par exemple dans le Notre Père. II s'agit de la fleur de la plante sauvage sunch'u (Encelia canescens) qui éclot, comme la pêche, à la période du Carême. Si les doctrineros se sont contentés de m'indiquer la syllabe commune entre le nom de cette fleur sunch'u et le substantif suti, «nom», plusieurs autres objets auraient pu évoquer le même mot, et ce de façon moins approximative.

En conséquence, en cette période vouée au recueillement et aux sacrements ${ }^{22}$, où toutes les pensées doivent être dirigées vers Dieu, le choix de cette fleur qui prolifère précisément à cette époque-là partout alentour n'est certainement pas fortuit. L'odeur, la couleur, les contours d'une fleur renvoyant au nom de Dieu bordent les chemins et les champs parcourus durant cette période de doctrine. De même, la sensation tactile que procure la caresse d'un textile ou la préhension de la laine livre des connaissances sans que celles-ci ne soient explicitement formulées. Tout en mémorisant, les enfants ont en effet l'habitude de toucher la boule de laine non tissée équivalant au péché ou de caresser les arcs de laine rose renvoyant à la sainteté. Les couleurs même de la laine donnent aussi un indice sur la révérence propre à certains termes ou concepts. Ainsi, si un bâtonnet de laine blanche renvoie au jour ou à l'année ${ }^{23}$, un autre de couleur bleu-vert indique le mot «vivant» (kawsaq) ou le verbe «ressusciter, revivre» (kawsarimpu- 24). Ailleurs, une croix formée de plusieurs brins de laine multicolores se réfère à «Dieu» tandis qu'un simple bâtonnet, également multicolore, indique le «règne» du Seigneur (dans la phrase «que ton règne vienne... »). Au $x v l^{e}$ siècle, le terme qhapaq signifiant «munificent, généreux (titre donné aux seigneurs puissants) a été choisi pour traduire le «règne» («être qhapaq ", c'est-à-dire être en capacité de donner des biens à une clientèle). Ainsi, il est possible que la laine multicolore évoque les riches vêtements colorés que, jusqu'à il y a quelques décennies, portaient les qhapaq. Dans les communautés, ceux-ci étaient les riches éleveurs, ayant beaucoup de bétail et pouvant donc obtenir, en échange de viande et d'engrais animal, la force de travail des familles sans bétail. Dans les Andes, la richesse est en premier lieu celle que donne le bétail, et l'association de la laine et du concept de qhapaq apparaît donc ici assez logique ${ }^{25}$.

La matière employée pour confectionner les rezos fonctionne à la manière de classificateurs, ces mots ou morphèmes associant les choses de la nature et les artefacts à des classes spécifiques. Ces classes forment des groupes sémantiques qui diffèrent en fonction des langues. Elles s'ordonnent suivant des caractéristiques formelles, l'usage ou toute autre propriété accordée aux objets par une culture donnée. Dans les transcriptions boliviennes, les matériaux donnent donc à voir une façon de constituer ces ensembles. Expérimenter les mots par une matière, des couleurs et des formes, c'est en intégrer directement le sens.

Les sens se conjuguent dans une expérience multisensorielle, voire synesthésique, dans la mesure où les mots sont vus, les images entendues, les couleurs et les formes éprouvées ou anticipées. Une fois la leçon terminée,
21. Plus rarement, le «nom» de Dieu peut être évoqué par un bâtonnet enroulé dans de la laine jaune, associant la préciosité de ce matériau à la couleur de la fleur sunch'u évoquée plus haut.

22. Cette période est propice à la confession mais aussi au baptême et à l'eucharistie, grâce au déplacement plus régulier du prêtre qui réside dans le chef-lieu et effectue plusieurs messes pour préparer Pâques.

23. Au mot "année" correspond un petit bâton auquel est simplement noué un fil de laine blanche. Le verbe attacher est en effet identique au mot qui désigne l'année solaire, wata.

24. Kawsarimpu-, "ressusciter» ou littéralement "d'un coup, se mettre à vivre ", est associé à un infixe dynamisateur -riet à un orientateur d'actance - pu.

25. Je remercie vivement César Itier de m'avoir notifié l'origine initiale de ce terme et d'avoir suggéré cette analyse. 
26. Au sujet de la question des valeurs et de leur expression, on se référera au stimulant article d'Angé 2016. L'auteur étudie précisément la façon dont des artefacts tricotés miniatures, vendus dans des marchés de villages nord-argentins, renvoient à différentes valeurs revendiquées par les communautés indigènes sans pour autant qu'elles soient formulées verbalement.

27. Enseigner, rappeler et émouvoir sont les fonctions essentielles des images telles que décrites par saint Thomas d'Aquin. Les atouts de l'image font l'objet de nombreux commentaires à l'époque moderne, dont les plus éloquents sont certainement ceux de Richeome (1597). Voir Dekoninck 2005. après l'avoir exploré de ses yeux et de ses mains dans sa version miniature, l'élève arpente un paysage grandeur nature. L'engagement du corps est donc partie prenante d'une religion faite de gestes et d'actions ordinaires, laquelle s'articule sans heurt avec le quotidien d'hommes associant indistinctement le faire au croire. Plus qu'un simple spectateur ou qu'un élève attentif, le catéchumène participe d'une croyance qui s'apparente davantage à un mode de vie qu'à une profession de foi dans laquelle il ne se reconnaîtrait pas forcément. Car cette pratique, autrefois encouragée par les prêtres, est aujourd'hui passée dans les mœurs et renouvelée chaque année. Et plutôt que de compléter les leçons des catéchistes ou les sermons du prêtre élaborés en espagnol, à l'église de la ville et un missel en lettres alphabétiques à la main, les autorités locales des communautés tendent même à rendre la pratique des rezos en trois dimensions obligatoire. D'une certaine façon, il apparaît en effet que l'élaboration, l'usage et même la destruction des rezos font apparaître les valeurs autour desquelles se reconnaît la communauté indigène ${ }^{26}$.

\section{Valeurs communautaires et contraste}

La fabrication de rezos tridimensionnels illustre le fait que l'apprentissage des prières et leur récitation n'est pas qu'une question de foi ou d'imaginaire. Non seulement les signes sont vus, lus et touchés, mais ils renvoient à des sensations liées à des périodes précises de l'année. C'est pourquoi une approche pragmatique éclaire ce processus d'enseignement pris dans un contexte d'action éminemment sensoriel. En outre, toutes les activités quotidiennes et essentielles à la survie du groupe alternent avec des activités exceptionnelles, pleines d'émerveillement ou de surprise. Le temps du Carême est sans doute le plus "à part" pour les catholiques: cette période de jeûne et d'abstinence alterne avec les fastes célébrations de Pâques. Or, dans la municipalité de San Lucas, le contraste sensoriel inhérent à ce rituel catholique se retrouve à différentes échelles, et tout d'abord dans le rapport à l'image.

\section{Absence et présence de l'image}

Les images enseignent, rappellent mais émeuvent aussi ${ }^{27}$. Mon hypothèse est que les images qui entrent dans la composition des rébus jouent un rôle mnémotechnique dans la mesure où elles sont incongrues et surprenantes. Comme le rappelle Quintilien, Cicéron préconisait, pour entraîner sa mémoire, de "faire le choix [...] d'images qui expriment quelque action, qui soient vives, caractéristiques, telles enfin qu'elles viennent au-devant de l'esprit, et le frappent incontinent» (Quintilien 1865: 417). Telles les «images agissantes» (imagines agentes) de l'Antiquité employées dans le cadre des arts de la mémoire, les rébus sont bien des images frappantes. Placées au cœur des textes doctrinaux, elles interfèrent avec les images plus sages du discours religieux. Qu'il s'agisse d'une pierre à moudre, de mandibules ou d'un morceau de tissu, toutes jouent sur le décalage puisqu'elles côtoient la Vierge, la croix et autres figurines de saints. Elles permettent donc de fixer plus aisément des termes par ailleurs difficiles à imager, voire si abstraits qu'ils sont irreprésentables. En provoquant le rire, elles sont même retenues avant les autres. Et c'est d'ailleurs un sourire aux lèvres que les adultes 


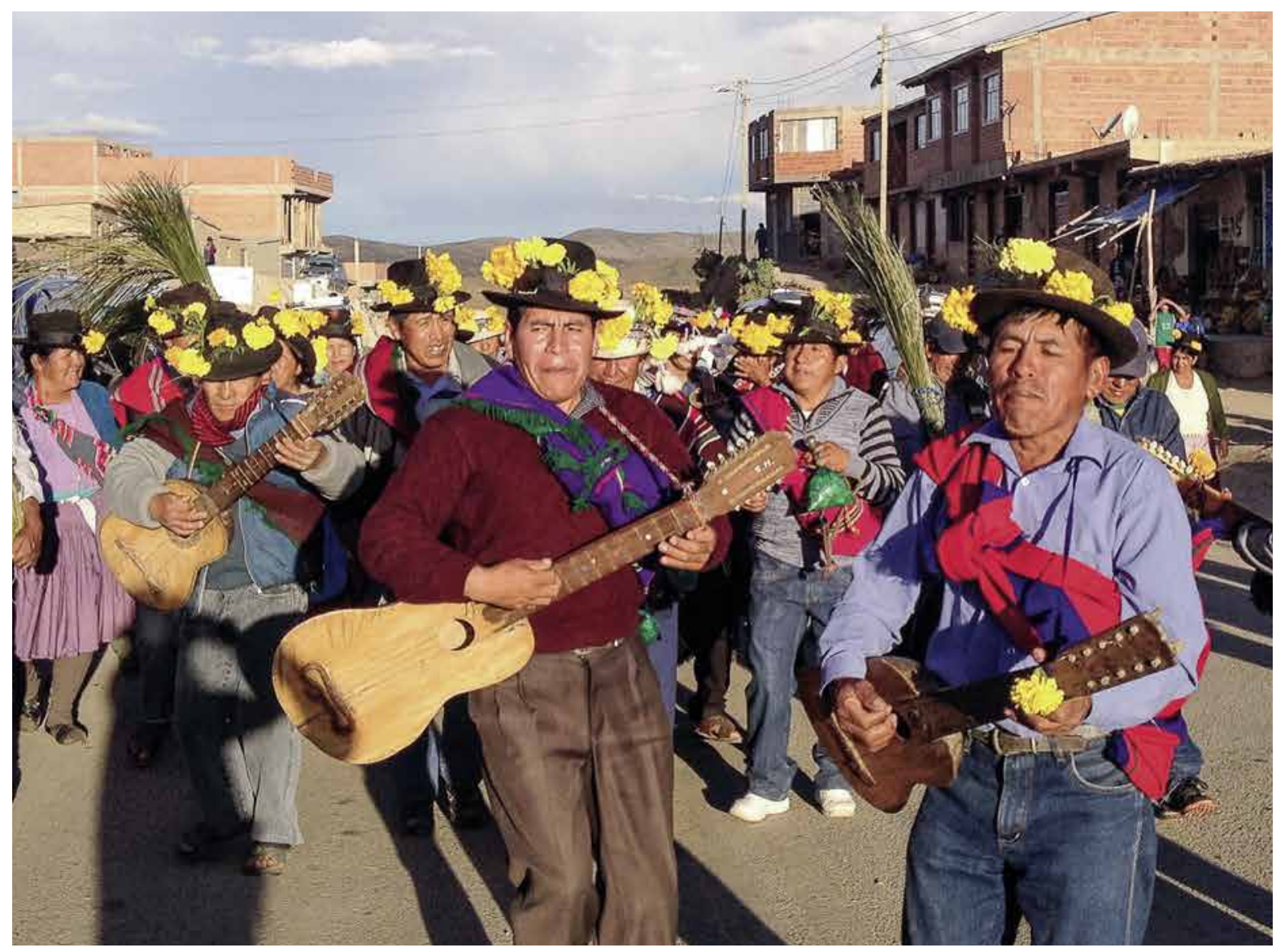

et les enfants expliquent, en priorité, le sens des objets entrant dans la composition de rébus: «Regarde, tu vois, ça c'est un chien, qui quand il aboie, fait "qan!" 28 ."

Mais, au-delà, ces images partagent leur caractère incongru avec le mode de communication iconique lui-même. Ne serait-ce que par leur aspect, ces textes tridimensionnels se singularisent fortement de ceux rédigés sur papier. Sortes de livres pop-up en argile, leur forme s'éloigne de ce que I'on associe traditionnellement à l'écrit. En outre, les dispositifs d'apprentissage du catéchisme sont annuellement créés dans des espaces où l'image est habituellement absente. Les humbles chapelles des communautés indigènes ne possèdent ni retables, ni vitraux, ni peintures. II y a peu d'affiches dans les rues, les habitants ont rarement une télévision, encore plus exceptionnellement un ordinateur, et peu de livres et de journaux circulent. Le Carême en Bolivie semble ainsi constituer un moment de forte iconicité, durant lequel les images sont multipliées, où l'on agit par l'image, les images devenant également agissantes par leur caractère d'exception.

Notons que ce contraste existe sous d'autres formes pendant le Carême, ailleurs et à d'autres époques. Les enjeux de visualité sont en effet d'une haute importance pendant le cycle de Pâques. Depuis le Moyen Âge, les images du Christ en croix prohibées sont révélées de façon spectaculaire le dimanche de Pâques. Ces pratiques médiévales faisant alterner occultation et monstration (Dittmar 2015: 94-95) ont encore cours fig. 8

Célébrations de Pâques lors du Samedi saint, communauté de Padcoyo, 2017. Photo Bérénice Gaillemin.

\section{En quechua,}

cette onomatopée est homophone du pronom personnel «lui». 
aujourd'hui dans différentes localités, en Europe et en Amérique. De nos jours, en Bolivie, si les images sont donc inexistantes au quotidien, elles se révèlent nombreuses pendant le Carême. En revanche, elles sont confinées dans des édifices sombres et au sein de dispositifs propices à la concentration et à la récitation de prières. De même, les prières sont récitées toute la nuit et dans une totale obscurité lors de la veillée pascale. Et ce n'est que le lendemain que les rares statues de l'église du chef-lieu sont portées en procession et au grand jour, moment d'une intensification sensorielle à la fois visuelle et sonore. D'ailleurs l'expression «Pâques fleurie» renvoie directement aux festivités de Pâques où des chants et des danses seront effectués, mais aussi où des fleurs seront fichées dans les chapeaux des participants.

\section{Humiliation et murmure, fierté et cri}

L'aspect de ces textes tridimensionnels, placés sur le sol, n'est certainement pas anodin. Leur fabrication comme leur usage imposent au corps une posture pénible, un exercice d'humilité. En effet, l'homme s'humilie littéralement en les lisant, il doit se rapprocher de la terre comme le requiert l'attitude de pénitence dont s'assortit le Carême. L'aspect sonore entre aussi dans la composition d'une ambiance riche de contrastes. Les prières sont en effet prononcées à la fois rapidement, sans articulation appliquée, mais elles sont aussi parfois susurrées. Elles sont également entonnées, scandées sur une mélodie qui est identique dans toutes les communautés. Les phrases y sont coupées de façon arbitraire pour correspondre à une sorte de mélopée.

Or cette période d'humiliation et de murmure ou chants discrets entre en alternance annuellement avec celle de Pâques où les prières sont littéralement criées à tue-tête lors de défilés. C'est d'ailleurs par le verbe «crier ", gritar qu'est décrite la procession finale, celle où le doctrinero de chaque communauté - «celui qui va crier » - marche fièrement en tête de ses ouailles. Tous en chœur, d'une seule voix étourdissante, ils hurlent les prières péniblement apprises pendant plusieurs jours ou semaines. Et c'est à ce moment que le chuchotement, parfois le brouhaha des heures d'apprentissage, se convertit en des chants retentissants qui se font écho, s'entremêlent, et forment une seule et même cacophonie joyeuse. Ce cri proféré ensemble, entre communautés mais également avec les métis de la ville chef-lieu, contraste par ailleurs avec la situation géographique et orographique de la région où les villageois sont dispersés et souvent isolés au sein de petits hameaux (entre 50 et 500 habitants, qui comptent parfois à peine dix familles).

\section{Paysages miniatures pour la Pachamama}

Convenons-en, la simple lecture de textes alphabétiques implique un effet gigantisme/miniature si l'on considère les caractères latins dans leur extrême petitesse (Stewart 1993). Ici, les petits «caractères» miniaturisent en outre diverses activités quotidiennes sur lesquelles enfants et adultes se penchent pour les observer dans le détail.

Ainsi, les disques d'argile peuvent être conçus comme des paysages miniatures, eux-mêmes imbriqués dans un impressionnant paysage. En effet, à plus grande échelle, l'église et la communauté prennent place dans un environnement où les sillons creusés par les pluies diluviennes de la saison humide côtoient des vallées montagneuses situées à plus de trois mille mètres d'altitude et où l'homme expérimente quotidiennement sa petitesse face à des forces qui le dépassent. 
Ce jeu d'emboîtement des espaces les uns dans les autres évoque le processus de démultiplication d'un objet à l'intérieur de lui-même, comme celui de structures architecturales suivant le principe des poupées russes ou de l'oignon (Golsenne 2009).

La terre où se déploie ce paysage est le domaine de la Pachamama, la déesse terre. Celle-ci est à la fois la mère nourricière et le sujet de toutes les attentions, source de tous les bienfaits. Or les objets - que l'on ne saurait désormais qualifier encore de logogrammes ou de phonogrammes - s'apparentent à une offrande, ou plus exactement à un vœu se référant aux activités de subsistance telles que la construction en argile, les récoltes de pêches, l'entretien des cheptels et le travail de la laine. II est ainsi possible de voir en eux le désir des habitants de projeter leur propre répétition, la continuité de ces mêmes activités, leur régénération étant implicitement souhaitée par le seul acte de les offrir à la terre sur laquelle ils se trouvent ${ }^{29}$. L'usage de l'argile s'assortit d'ailleurs de libations, de l'alcool étant versé sur chaque rezo au moment de leur élaboration et avant leur destruction. En miroir de ce don à la terre, les responsables de la Doctrina, après avoir terminé de modeler les rezos et avant de les détruire, consomment de l'alcool et mastiquent ensemble des feuilles de coca.

\section{Matérialisation d'un équilibre}

Au-delà de l'enseignement d'un corpus de textes, l'enseignement des rezos en quechua s'apparente à une silencieuse, tamisée, sourde et subtile «leçon de choses» ou de vie dans la communauté et plus globalement d'être au monde. Cet enseignement passe par l'expérience esthétique et celle-ci, me semble-t-il, a bien vocation à confirmer l'existence de groupes sociaux collectifs. Le paysage, dirions-nous, est offert en même temps qu'est demandé son renouvellement à la mère qui l'engendre. Et, pour ce faire, il doit réunir certaines caractéristiques: se révéler à la fois équilibré, complet et éphémère, le tout supposant une élaboration, un usage et une destruction placés sous le signe du collectif.

On pourrait en effet se demander ce qui caractérise un rezo bien fait, quelles en sont les caractéristiques esthétiques (dans tous les sens du terme), c'est-à-dire ce qui le rend efficace aux yeux des habitants et, d'un certain point de vue, ce qui en fait quelque chose de «beau » (Jeudy-Ballini 1999). Dans la mesure où ce terme n'est pas employé, rappelons en premier lieu une chose: la virtuosité du maestro s'exprime dans l'art de bien récolter l'argile, de former le disque de façon régulière, mais surtout d'en apprécier au mieux le diamètre. Celui-ci doit en effet être en adéquation avec la longueur du texte à transcrire. Pas de repentir possible: si la taille du disque est trop petite, la totalité des figurines nécessaires à la transcription du texte en question ne pourra s'y loger. À l'inverse, puisque les premières figurines sont disposées sur le bord du disque tandis que la spirale se termine en son centre, si le «scribe» a pensé sa page ronde à la hausse, alors les objets pourront y trouver chacun leur place mais un trop grand vide sera laissé au milieu. Le rezo sera certes «complet» (cabalito, «tout complet») mais mal disposé et les espaces seront trop importants pour que la transcription soit jugée régulière et harmonieuse. L'anticipation du texte joue donc dans l'appréciation de la forme, occasion pour le maître de montrer sa virtuosité et de faire part de son expérience.
29. À propos de l'idée de coactivité mimétique dans un cadre rituel, voir Pitrou 2016. 

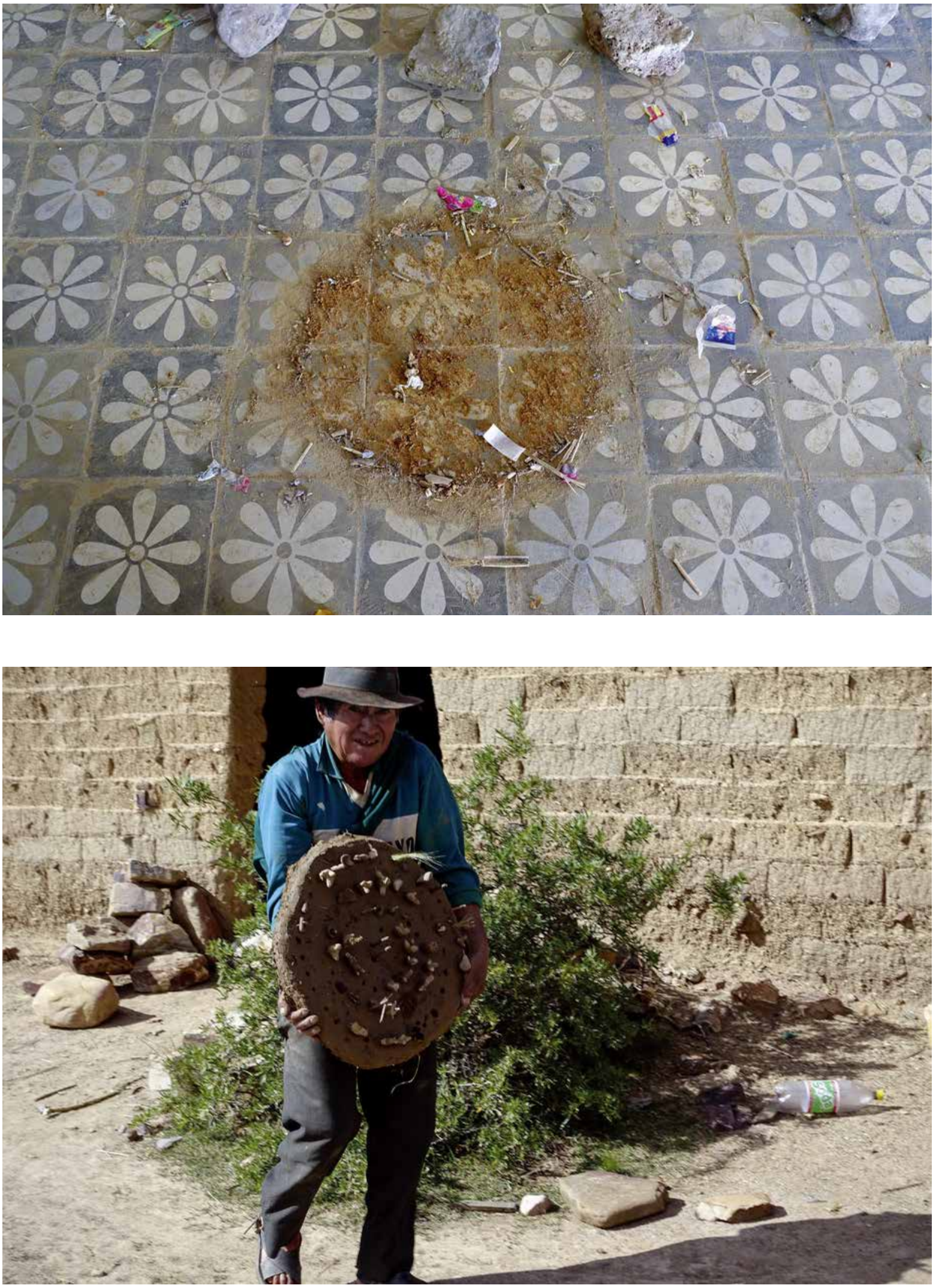

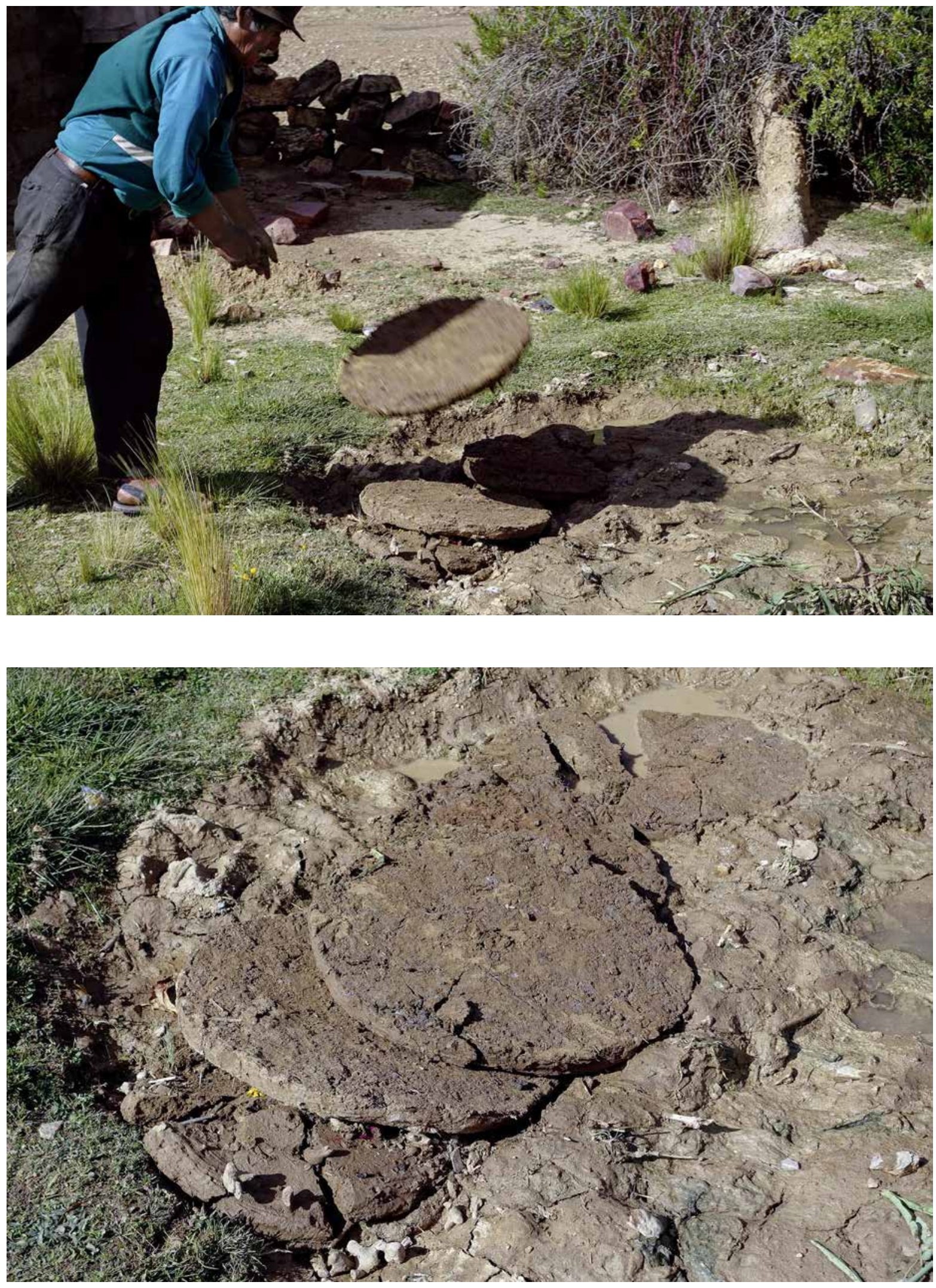
double page précédente

fig. 9, 10, 11 et 12

À Pâques, la destruction des rezos s'effectue par l'autorité supérieure de la communauté après libations et consommation d'alcool. Communautés de Tirahoyu et Padcoyo, 2015. Photos Pierre Déléage.
L'aspect collectif de cette élaboration semble néanmoins beaucoup plus important que cette individuation en rapport à un arrangement des objets finalement peu commenté. En effet, les figurines sont modelées à plusieurs. II ne s'agit en l'occurrence pas d'un seul scripteur mais de tout un groupe réuni pour l'occasion dans l'édifice choisi pour l'enseignement de la Doctrina. Les autorités des communautés responsables modèlent les figurines, accompagnées parfois d'autres habitants et enfants participant à la confection des prières. Certaines figurines peuvent être élaborées à la chaîne par une même personne, chargée par exemple d'effectuer l'ensemble des nombreux suffixes pluriel qui seront utilisés (en l'occurrence les petites pierres à moudre modelées en argile). Les signes modelés sont ensuite placés sur le disque par le doctrinero qui connaît la syntaxe. Les enfants se réjouiront plus tard de montrer le charango (sorte de petite guitare), le chien ou la poule qu'ils ont confectionnés.

L'apprentissage qui suit est lui aussi collectif. II est même obligatoire puisque chaque famille appartenant aux communautés du groupe rotatif organisateur de l'enseignement est priée, sous peine d'amende, d'envoyer un ou plusieurs de ses membres. Le nombre de jours de présences minimum varie en fonction des communautés et de la disponibilité du maestro. Ainsi Yolanda, 19 ans, bien qu'elle travaille en Argentine comme couturière, vient rendre visite à ses parents pour Pâques et doit s'astreindre à quatre jours complets de leçons. Dans le cas contraire, en compensation - et toujours à la communauté -, la famille versera une somme d'argent ou offrira une charge de bois de chauffage. Cet argent, comme celui récolté pour payer le maître et engager les musiciens jouant lors des célébrations de Pâques, sera lui aussi utilisé par et pour tous. Le bois servira quant à lui à faire le feu utilisé lors de la veillée pascale réunissant tout le village. Par ailleurs, à l'apprentissage ensemble, correspond le chant qui complète les leçons: il est également exécuté d'une seule voix et de façon collective, sous forme de répétitions entre un récitant-chanteur solitaire et le groupe des élèves en chœur.

Enfin, à l'issue de l'apprentissage, c'est encore en groupe que les rezos sont détruits. Après avoir récité une dernière fois chacune des prières avec le maître, les élus de l'année annoncent qu'il est désormais l'heure de casser le tout. Et tandis que quelques figurines sont retirées des disques afin d'être conservées pour l'année suivante (il s'agit des figurines ou objets les plus «précieux», en l'occurrence ceux qui sont élaborés avec de la laine), les disques sont jetés devant la porte de l'édifice, puis acheminés dans une brouette jusqu'à la décharge la plus proche. À l'instar des rituels associés à la fabrication des masques Sulka de Papouasie-Nouvelle-Guinée, l'acte de faire, défaire et refaire les rezos inscrit l'existence de ces objets dans «un cycle infini de disparitions et de réapparitions, comme s'ils renaissaient constamment de leurs cendres » et autorisaient à vivre à la fois dans la reproduction et la continuité (Jeudy-Ballini 1999 et 2015).

La pratique de l'enseignement des rezos s'inscrit dans un cycle qui est à la fois religieux, politique et agricole. La destruction des rezos met un point final à la période de l'enseignement de l'année et marque le début des célébrations pascales. II ne s'agit guère pourtant d'un iconoclasme car la destruction des disques d'argile s'impose pour ainsi dire d'elle-même. En effet, les disques en matériau friable s'altèrent facilement et en quelques semaines, d'autant plus qu'ils ont été sollicités de façon répétitive par la baguette 


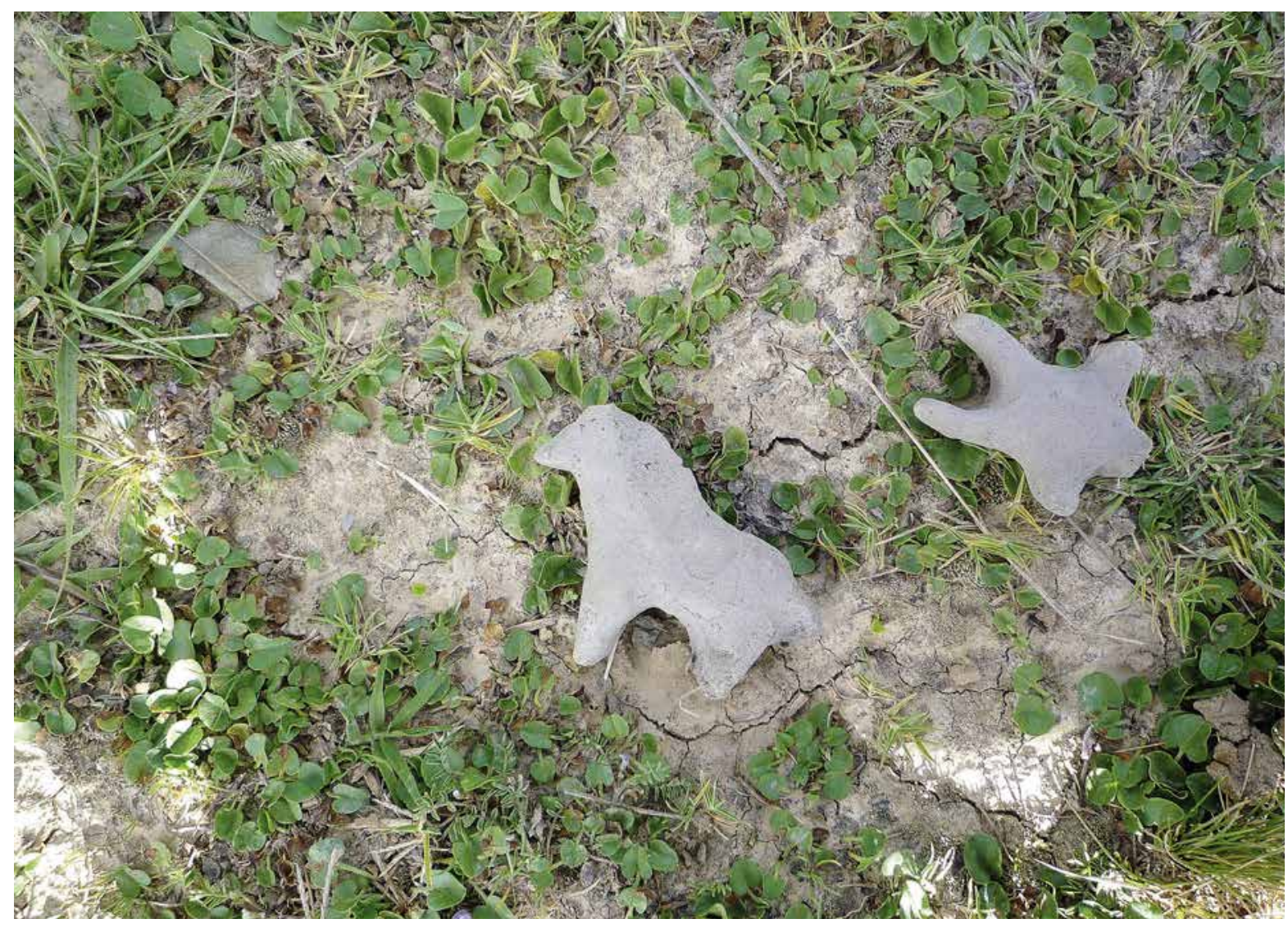

du maître et des apprenants. Les figurines ont aussi parfois été touchées, cassées ou brisées, malmenées par les enfants curieux, parfois avides d'en faire leurs jouets; les fleurs se sont fanées, les épis et les plantes ont séché. En somme, ce paysage miniature est saisonnier, et il convertit l'expression religieuse en une expérience véritablement sensationnelle, provoquant une sorte d'émerveillement et mobilisant périodiquement à la fois les corps des pratiquants, leurs gestes, leurs sens et leurs émotions (Meyer 2015). Sa destruction, accompagnée de gestes rituels, invite à le renouveler lors du prochain Carême de la même façon que les fleurs sunch'u envahiront à nouveau le paysage, que les pêches pèseront encore sur les branches des arbres et que le collectif sera lui aussi appelé à se rassembler.

fig. 13

Quelques figurines

jonchent le sol pendant encore plusieurs mois:

ce sont les pièces en double ou cassées fabriquées en groupe lors du Carême, communauté de Tirahoyu, 2013. Photo Bérénice Gaillemin. remerciements

Je remercie chaleureusement

Isabel Yaya et Pierre Déléage avec qui j'ai partagé mes premiers terrains, ainsi que Danièle Dehouve, Charles Stepanoff Anouk Cohen, Samir Boumediene et César Itier pour leurs corrections et précieux commentaires. 


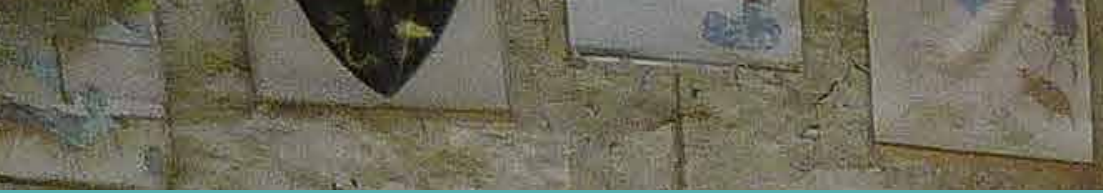

Bibliographie

\section{Angé, Olivia}

2016 « Miniatures in Mesoamerica and the Andes: Theories of Life, Values, and Relatedness", Journal of Anthropological Research 72 (4): 408-415.

1979-1986 Gérard Blangez (dir.), Ci nous dit: recueil d'exemples moraux, t. I. Paris, Société des anciens textes français.

\section{Céard, Jean et Margolin, Jean-Claude}

1986 Rébus de la Renaissance:

des images qui parlent.

Paris, Maisonneuve et Larose.

\section{Dehouve, Danièle}

2011 «Analogía y contigüidad en la plegaria indígena mesoamericana", Itinerarios 14: 153-184.

2014 "Flores y tabaco: un difrasismo ritual », Revista Inclusiones 1 (2) : 8-26.

\section{Dekoninck, Ralph}

2005 Ad Imaginem: statuts, fonctions et usages de l'image dans la littérature spirituelle jésuite

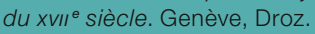

2011 "Une pédagogie jésuite de et par l'image: la "langue des images" du Père Steegius (1647)", in Annie Renonciat (dir.), Voir/savoir la pédagogie par l'image aux temps de l'imprimé, $d u x v I^{\circledR}$ au $x x^{e}$ siècle. Rouen, CNDP : 173-180.

\section{Déléage, Pierre}

2013 Inventer l'écriture: rituels prophétiques et chamaniques des Indiens d'Amérique du Nord, $x v I^{e}-x I x^{e}$ siècles.

Paris, Les Belles Lettres.

\section{Dittmar, Pierre-Olivier}

2015 «Cachez ce saint que je ne saurais voir. Modifications de visibilité en contexte rituel à la fin du Moyen Âge ", Cahiers d'anthropologie sociale $11:$ 84-99.

\section{Golsenne, Thomas}

2009 "L'ornement est-il animiste? " in Histoire de l'art et anthropologie [en ligne], disponible sur: http://actesbranly.revues.org/282 (consulté le 13 juillet 2017).

D'argile, de fleurs et de laine. Catéchismes multisensoriels dans les hauts plateaux boliviens. Par Bérénice Gaillemin

\section{Jeudy-Ballini, Monique}

1999 " "Dédommager le désir".

Le prix de l'émotion

en Nouvelle-Bretagne

(Papouasie-Nouvelle-Guinée) ", Terrain 32: 5-20.

2015 «Muséographier l'émotion esthétique? Réflexions à propos d'un exemple océanien ", THEMA. La revue des Musées de la civilisation $2: 45-54$.

\section{Lakoff, George et Johnson, Mark}

1985 Les Métaphores dans la vie quotidienne, trad. de l'américain par Michel De Fornel.

Paris, Les Éditions de Minuit.

\section{Meyer, Birgit}

2015 "How to capture the "wow"

R. R. Marett's Notion of Awe and the Study of Religion ", Journal of the Royal Anthropological Institute 22 7-26.

\section{Miranda Mendoza, Juan}

1988 Catecismo. Diuswan munanakuypi kawsanapaj. Sucre, Talleres Gráficos Tupac Katari.

\section{Miranda Rivera, Porfirio}

1958 "Quipus y jeroglíficos ",

Zeitschrift für Ethnologie 83 :

118-132.

\section{Pitrou, Perig}

2016 Le Chemin et le champ: parcours rituel et sacrifice chez les Mixe de Oaxaca (Mexique). Nanterre, Société d'ethnologie.

\section{Quintilien}

1865 L'Art oratoire, in M. Nisard (dir.), Quintilien et Pline Le Jeune. œuvres complètes.

Paris, Firmin Didot Frères.

\section{Richeome, Louis}

1597 Trois Discours pour la religion catholique: des miracles, des saints, des images. Bordeaux, S. Millandes.

\section{Roudaut, Fanch, Croix, Alain et Broudic, Fanch}

1988 Les Chemins du paradis Taolennou ar baradoz,

photographies de Padrig Sicard. Douarnenez, Le Chasse-MaréeÉditions de l'Estran.

\section{Stewart, Susan}

1993 On longing: Narratives of the Miniature, the Gigantic, the Souvenir, the Collection. Durhan, Duke University Press.

\section{Yates, Frances A.}

1966 The Art of Memory. Chicago, The University of Chicago Press. page 46 et ci-contre Les dix prières à mémoriser. La première est le Notre Père, située à gauche de l'entrée de l'ancienne église de la communauté de Padcoyo, 2017.

Photo Bérénice Gaillemin.

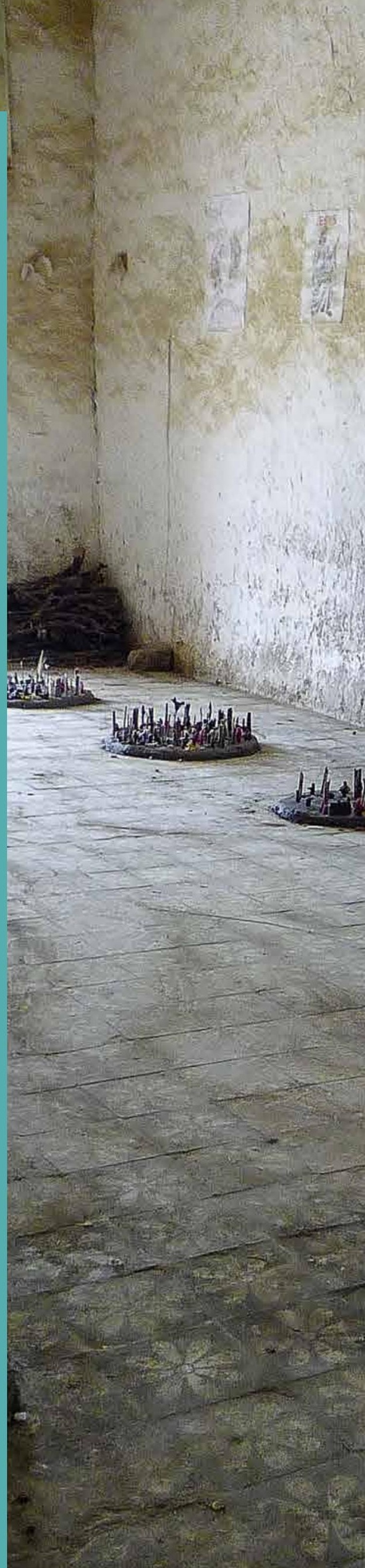


